\title{
Frequency of Recovered Orders at the Cardiac Care Unit
}

\author{
Ravanbakhsh Esmaeili, ${ }^{1}$ Masoumeh Bagheri Nesami, ${ }^{2}$ Asghar Nadighara, ${ }^{3}$ and Aghdas Taghizadeh ${ }^{1, *}$ \\ ${ }^{1}$ School of Nursing and Midwifery, Department of Medical Surgical Nursing, Orthopedic Research Center, Mazandaran University of Medical Sciences, Sari, IR Iran \\ ${ }^{2}$ School of Nursing and Midwifery, Department of Medical Surgical Nursing, Pediateric Infectious Disease Research Center (MPIDRC), Mazandaran University of Medical \\ Sciences, Sari, IR Iran \\ ${ }^{3}$ Health Sciences Research Center, Mazandaran University of Medical Sciences, Sari, IR Iran
}

Corresponding author: Aghdas Taghizadeh, School of Nursing and Midwifery, Department of Medical Surgical Nursing, Orthopedic Research Center, Mazandaran University of Medical Sciences, Sari, IR Iran. E-mail: aghdastaghizadeh@yahoo.com

Received 2015 December 21; Accepted 2015 December 23.

\begin{abstract}
Background: Patients' safety is one of the basic concepts in health care systems and one of the major concerns of patient care. Medical errors are amongst the main factors that could threat patients' safety. At intensive care units, there is always the possibility of errors during various stages such as the ordering stage.

Objectives: The aim of this study was to determine the frequency of pharmacological and non-pharmacological recovered orders at intensive care units and its associated issues.

Patients and Methods: This study was a cross-sectional study based on pharmacological and non-pharmacological orders obtained from patients' records and daily record sheets at the intensive care units of Mazandaran heart center during year 2015. All orders from patients' records $(\mathrm{n}=1046)$, which included 29214 orders, were reviewed during a three-month period. Data was collected using a researcher-made checklist and included patients' demographic information and a medical checklist on the patients' conditions, name of the person who had recorded the order and the person who had discovered the recovered order, type of recovered pharmacological and non pharmacological orders and related factors. To analyze the data, statistical tests such as mean and relative frequency, contingency parts and chi-square test were applied using the SPSS 19 software.

Results: A total of 29214 pharmacological and non pharmacological orders, written in 1046 patients' records of six coronary care units were investigated. We found that $150(0.5 \%)$ recovered orders were documented. The results showed a significant relationship between the academic year of medical assistants and the recovered orders $\left(K_{2}=88.619, \mathrm{P}<0.05\right)$; most orders were recovered by second-year assistants.

Conclusions: According to this study, it is necessary to define a procedure for careful review of pharmacological and non pharmacological orders. In addition, it is recommended to use clinical pharmacologists and expert critical care nurses to allow the possibility of discovering wrong orders before putting them into practice.
\end{abstract}

Keywords: Pharmacological Recovered Orders, Non-Pharmacological Recovered Orders, Coronary Care Units (CCU)

\section{Background}

Maintaining patient's safety is one of the basic concepts in health care systems; managers and staff's efforts are very important in this regard (1-3). Medical errors obviously influence patients' safety (1). Despite all the efforts, medical errors are inevitable and according to investigations, they are still happening in the current health system $(4,5)$. Committing an error is an integral part of the human life. All the staff of health care teams, regardless of their skills, commitment and accuracy, may commit an error in performing professional tasks (4). Among different hospital wards, intensive care units are preventable in terms of human errors and inappropriate events are more considered in the health care system of these wards (6). One of the errors at intensive care units is pharmacological and non-pharmacological orders that are issued and written, but are recovered before or after performance ( 7 , 8). Some recovered orders are in fact among errors that will harm the patients in the case they are not recovered (8). Errors resulting from false orders and medical errors are the eighth common cause of patients' death in the US (4). Annually, 44 to 98 thousand patients are dying due to mistakes and errors in the US (9-12). Also statistics have shown that the number of deaths resulting from pharmacological and non-pharmacological errors are more than the number of deaths due to motorcycle accidents, breast cancer, Human Immunodeficiency Virus (HIV), poisoning and drowning $(3,13)$. According to the achieved results, from $4 \%$ to $17.7 \%$ of patients in the world are affected by adverse consequences arising from pharmacological and non-pharmacological errors (5). Costs of pharmacological and non-pharmacological errors have been reported as 
17 - 29 billion dollars (12-14); such costs are for compensation of adverse events resulting from pharmacological and non-pharmacological errors (9). The World Health Organization (WHO) has reported in 2009 that there are 10 million cases of morbidity or mortality due to unsafe health services around the globe (15). In addition, since medical unwanted errors affect one in every ten patients throughout the world, WHO has referred to it as endemic. Also a patients' safety national organization has recently provided some guidelines with the aim of promoting patients' safety (16). There is increased risk of committing errors at intensive care units during different stages of treatment such as writing orders; this is due to the nature of such wards such as appearance of emergency and critical situations, variety of medications and medication orders, different methods of drug delivery to patients, critically ill patients and their need for accurate care at all times (3, 17-19). Studies have shown that other factors such as the number of admissions, prescriber's work-load and their knowledge, are also effective factors on the occurrence of wrong orders (20-22). These recovered orders may be in the form of pharmacological recovered orders such as prescribing wrong drugs or non-pharmacological recovered orders such as diagnostic errors $(7,23,24)$. Nowadays, to reduce the incidence of errors, they have been used as an index for determining the level of patients' safety in hospitals (3). Results of studies have shown that errors are common at intensive care units (25) and there are 1.7 cases of pharmacological and non-pharmacological errors every day (17). Death and patient's critical condition are the adverse consequences of not recovering mistakes and pharmacological and non-pharmacological errors $(7,8)$. According to the available statistics, the outcomes due to pharmacological and non-pharmacological errors do not only harm the patients, but also cause physical and mental stress for the health care staff, patients' relatives, and even the society and reduce patients' satisfaction and trust $(26,27)$. Most studies regarding medical errors and their related factors $(24,28)$ have shown that nurses, doctors, clinical pharmacists and even the patients themselves play effective roles in recovering pharmacological and non-pharmacological errors and preventing their complications $(7,24)$. According to the available statistics, nurses are the first treatment and care force; they have an important role in the improvement procedure of critically ill patients that need careful and constant care. This is due to their constant presence at intensive care units. Therefore, nurses play an important role in performing duties related to patients' safety. The recovered orders can indicate the possibility of committing errors. Recovered errors show us how to prevent errors that can harm patients in the future. Therefore, studying errors and recovered or- ders are important.

\section{Objectives}

This study was designed and performed with the aim of determining the frequency of pharmacological and nonpharmacological recovered orders at the CCU to assess the frequency of recovered orders and to identify their related factors.

\section{Patients and Methods}

This study was a descriptive-analytical research that was done to determine the frequency of pharmacological and non-pharmacological recovered orders and their related factors at the CCUs of Mazandaran heart center during years 2014 to 2015. The research environment included six CCUs (heart surgery and CCU) of Mazandaran heart center. For performing the study, referred to the related center with a referral letter from their education provider and started the study after obtaining permission from the center manager. The orders available in the patient files and the recording sheet about the daily situation of the patients were assessed for three months through a census method in terms of frequency of pharmacological and non-pharmacological recovered orders and their related factors. Also the medical staffs (patient's doctor, the doctor in charge of the ward, assistants, nurses and head nurses) were the information source. The researcher assured the participants that all their information will be confidential. Two researcher-made checklists, which were designed by collecting texts and lecturers' views, were used as a tool for collecting data. Some questions were answered by observing the files and the record sheet of the patients' daily condition and some others were answered through interview and interaction with the order discoverer and issuer. Demographic and clinical features of the patients, the orders discoverer and issuer were collected in the first checklist. Nine questions were on demographic and clinical features of the patients including; age, gender, type of diagnosis and hospitalization ward, shift, day and duration and the number of pharmacological and non-pharmacological orders. The eight questions about demographic features of the order's issuer included; age, gender, type of expertise, education level, assistant's academic year, work experience, work experience at the current ward and the way of ordering. Also, this checklist included six questions on demographic features of the discoverer regarding age, gender, education level, job responsibilities, work experience at other wards and work experience at the current ward. Information on pharmacological and non-pharmacological 
recovered orders and their related factors at the CCU were written in the second part of this checklist and included 11 questions. pharmacological errors, name of the pharmacological group, changes in the recovered pharmacological orders, cause of wrong order, discovering the recovered orders before administration, working shift when the recovered order was issued and causes that led to the discovery of recovered orders. Checklists were prepared and given to 10 faculty members, specialists in heart surgery, heart, intensive care and nursing to investigate the validity, and their reforms were considered in the checklists. Also intra-observer agreement coefficient (kappa) was used for determining the tools reliability. The achieved agreement coefficient was reported over 0.7 , which indicated the reliability of the tool. The researcher attended the research location during three work shifts of morning, evening and night and assessed the issued orders in the file and the recording sheet of the patients' daily condition. In this study, at four of the CCUs, all of the orders were written traditionally and manually, while at two CCUs writing orders traditionally was combined with computerized recording. Recovered orders in this study were pharmacological and non-pharmacological orders written in the patients' file, which were identified, cancelled or recovered as a wrong order by the issuer or discovered before or after administration. In this study $\mathrm{P}<0.05$ was considered significant. The collected data were assessed and analyzed with the SPSS 19 software and descriptive statistical tests such as average, absolute and relative frequency, contingency and analysis such as chi-square test.

\section{Results}

Overall, 29214 pharmacological and nonpharmacological issued orders in the file and recording sheet of daily condition of 1046 patients hospitalized at different CCUs of Mazandaran heart center were assessed for three months. In total, 17215 pharmacological orders and 11999 non-pharmacological orders were issued by the issuers. The average and standard deviation of issued pharmacological and non-pharmacological orders during hospitalization in 1046 files and totally 29214 issued orders were $16 \pm 14$ and $11 \pm 7$, respectively. Among 29214 orders, 150 orders were recovered. Also, the results showed that there were 130 recovered pharmacological orders and 20 recovered non-pharmacological orders. Six hundred and seven files were related to male and 439 files were related to female patients. The average and standard deviation of the patients' age was $61.27 \pm 11.81$. Furthermore, $89.7 \%$ of the patients were hospitalized on working days and $10.3 \%$ of the patients were hospitalized on holidays. The mean of hospitalization duration was three days. Most of the patients were admitted during the evening shift (57.4\%), morning shift (33.1\%) and night shift (9.6\%). The average age and standard deviation of the issuers of the recovered orders were $34.41 \pm 5.49$ years old (the age range was 30 to 63 years old). Overall, $55.3 \%$ of the recovered orders were issued by males and $44.7 \%$ of the orders were issued by females. Moreover, $84.7 \%$ of the orders were issued by medical assistants, $10.7 \%$ by specialists and only $4.7 \%$ by subspecialists. The heart internal department had issued 88.7\% of the recovered orders. Average and standard deviation of work experience of the recovered orders issuers were $35.11 \pm 47.64$ months (5 to 240 months). Sixty-eight percent of the recovered orders discoverers were female and $32 \%$ were male. Also $67.7 \%$ of administrative orders discoverers had a PhD degree and 31.3\% had MA. The average and standard deviation of work experience at the ward of the order discoverers were $73.24 \pm 54.44$ months (five to 180 months). There was a significant statistical relationship between work shift of the issuers and the recovered orders at the CCU $\left(\mathrm{K}_{2}=26.040, \mathrm{P}<0.05\right)$, so that most of the recovered orders (46\%) were issued in the morning work shift, followed by the night work shift with 60 cases (40\%). Also the least number of recovered orders (14\%) were issued during the evening shift. There was a significant statistical difference between the academic year of medical assistants who were issuing the orders and the recovered orders at CCUs $\left(K_{2}=88.619, \mathrm{P}<0.05\right) ; 69.3 \%$ of the recovered pharmacological orders were issued by the assistants of the second year (Table 1 ).

Table 1. The Academic Year of Medical Assistants That had Issued Recovered Orders ${ }^{\mathrm{a}}$

\begin{tabular}{ccc}
\hline \multirow{2}{*}{ Assistant Academic Year } & \multicolumn{2}{c}{ Recovered orders } \\
\cline { 2 - 3 } & Number & Relative Frequency \\
\hline Second Year & 87 & 69.3 \\
Third Year & 38 & 29.9 \\
Fourth Year & 1 & 0.8 \\
\hline
\end{tabular}

${ }^{\mathrm{a} C h i}-2$ statistical test: $\mathrm{K}_{2}=88.619 ; \mathrm{P}[\mathrm{U}+02 \mathrm{C} 2] 0.05$.

Overall, 55.3\% (83 orders) and $44.7 \%$ (67 orders) of the orders were issued, respectively by males and females; there was no significant statistical relationship between gender of the issuer and the recovered issues $\left(\mathrm{k}_{2}=1.707, \mathrm{P}\right.$ $>0.05$ ) (Table 2).

There was a significant statistical difference between order discoverers' profession and the recovered orders at CCUs $\left(K_{2}=19.440, \mathrm{P}<0.05\right)$. One hundred and two orders were discovered in this study; $68 \%$ were issued by doctors and $32 \%$ by nurses. Most of the recovered orders involved the cardiovascular drug group (46.2\%). Also $22.3 \%$ were related to anticoagulant and thrombolytic factors and $12.3 \%$ 
Table 2. The Relationship Between the Issuers' Gender and Pharmacological and Non-Pharmacological Recovered Orders ${ }^{\mathrm{a}}$

\begin{tabular}{lcc}
\hline \multirow{2}{*}{$\begin{array}{l}\text { Order Issuers Gender } \\
\text { Number }\end{array}$} & \multicolumn{2}{c}{ Recovered Orders } \\
\cline { 2 - 3 } Male & Relative Frequency \\
\hline Female & 63 & 55.3 \\
\hline
\end{tabular}

${ }^{\mathrm{a}}$ Chi-2 statistical test: $\mathrm{K}_{2}=1.707 ; \mathrm{P}>0.05$

were related to antibiotics. This is while the least recovered orders were related to the central nervous system (CNS) drugs. One hundred and forty-eight (98.7\%) recovered orders were written by the issuers. The average age and standard deviation of patients, who had been issued recovered orders was $63.46 \pm 13.59$ years old. Also there was a significant statistical difference between the patients' age and the recovered orders at the CCUs in the mentioned study $\left(\mathrm{K}_{2}=40.533, \mathrm{P}<0.05\right)$. Most of the recovered orders were for above 70 -year-old patients (31.3\%).

\section{Discussion}

The results of our study showed that 150 recovered orders were issued and corrected during three months. There were 130 (86.7\%) recovered pharmacological orders and 20 (13.3\%) non-pharmacological orders, while in a similar study by Rothschild et al. (2006), performed during 147 days, 142 recovered medical errors were reported (8). Also in another study by Rothschild et al. (2010) from the USA, there were 504 recovered orders that is $7.8 \%$ for every patient and 2.9\% for every issued pharmacological order (29). In a study by Vazin et al. (2014), 707 (68.5\%) medical errors were reported at different pharmacological stages; among them, 217 errors (21.1\%) were during the stage of issuing the order (30). In the study of Stasiak et al. (2013), 99 errors (3.2\%)(31), and in the study of Vessal (2009) 86 errors (10.5\%) of recovered orders were calculated and reported (32); the cause of contradiction in the results of recent studies with the present study can be due to assessment of recovered orders during different stages of drug prescription procedure, difference in the sample volume, difference in patients' demographic features, type of hospitalization, difference in type of wards and using or lack of using computerized recording system in the case of issuing orders. In this study, most of the recovered orders were issued during the morning shift, which is consistent with the results of the study of Vazin et al. (2012) (33). However, in the studies of Mohsenzadeh (2009), Kozer (2002) and Zeraatchi (2013) et al. increased issuing of recovered orders and more pharmacological errors were reported during the night work shift (34-36), also in the study of Stasiak et al. (2013), more than half of the errors in issuing pharmacological orders had occurred in the evening, which are not consistent with the results of the present study (31). The cause of this contradiction is the difference in the system and the way of admitting patients; in the patient medical centers, most of the patients are admitted by the assistants and the orders are written by them in the patients' file, therefore the morning shift is very busy. There was a significant statistical difference between the academic year of the medical assistants, who issued the order, and the recovered orders in different parts of the CCU. So that assistants of the second year issued $69.3 \%$ of the recovered orders; results of this study are similar to the study of Ryan et al. (2014) in Scotland (21). However, in the study of Seden et al. (2012), it was clear that there was no significant difference between assistants' academic year in issuing orders and there was no remarkable difference between novice doctors and medical assistants of higher years (37); the cause of the difference of these results with the present study can be due to the difference in the protocols of patients' admission and the way of writing orders in different countries. There was no significant statistical difference between issuers' gender and the recovered orders in the present study; results of this study are consistent with the results of the study of Shamsaii et al. (2010) (1). However, in the study by Aala et al. (2014), it was reported that most of the issued pharmacological orders had been done by males (38), which is in contrary with the results of the present study and it could be due to the lack of difference in gender of the recovered orders' issuers in our study. There was a significant statistical difference in the present study between age of the patients and the recovered orders; this result is consistent with the findings of the studies done by Mousavi (2012), Zeraatchi (2013), Dabaghzadeh and Stasiak et al. (31, 35, 39, 40). This is while in the study done by Mohsenzadeh et al. (2009), it was clear that most of the pharmacological errors were related to younger than two-year-old children (34). The cause of this difference could be due to children's sensitivity and the difference of the issuers in terms of specialty in the two studies. The present study showed that the most common form of issuing recovered orders at the CCU was the written form. Also results of studies of Stasiak (2013) and Evans et al. (2009) showed that pharmacological recovered orders are not general in the oral form; results of these studies are similar to the present study $(31,41)$. There was a significant statistical difference between orders discoverers' profession and recovered orders, which is consistent with the findings of Osmon (2004) and Rothschild et al. (2005) studies $(42,43)$; most of the wrong orders were discovered and recovered by doctors. Most of the recovered orders were related to cardiovascular drugs and the 
least were related to central nervous system drugs. In the study done by Stasiak et al. (2013), it was reported that the most common recovered orders included the cardiovascular drug group (21.2\%), followed by neurological drugs (10.1\%) and inhaled drugs (9.1\%)(31), which are similar to the results of this study. Recovered orders were issued mostly by first year assistants, therefore, it is recommended to have a detailed review of medical, pharmacological and non-pharmacological issued orders before administration and to design and perform a supervision and training system regarding controlling issued orders by the assistants. Some strategies are recommended for maintaining and promoting patients' safety in health services, increasing patients and their relatives' trust and satisfaction and decreasing the cost of country health system; these strategies include using clinical pharmacologists and nurses specialist in intensive care; these strategies increase the possibility of discovering and diagnosing wrong orders before performance. Also it is recommended to use randomized observational studies in the future studies for achieving accurate statistical frequencies regarding recovered orders at CCUs.

\section{Acknowledgments}

It is necessary to thank and appreciate the Mazandaran Medical Sciences University research and technology deputy for their support and approval of this project. Also we thank and appreciate the cooperation and help of the authorities, doctors and nurses working at the CCUs of Mazandaran heart center.

\section{Footnote}

Funding/Support: This manuscript was derived from a research study and an intensive care nursing MA thesis.

\section{References}

1. Shamsaii M, Faraji O, Ramazani A, Hedaiati P. The viewpoints of zabol's general practitioners about medical errors in 2010. Psychiatr Q. 2012;10(4):31-6.

2. Valizadeh F, Ghasemi SF, Nagafi SS, Delfan B, Mohsenzadeh A. Errors in medication orders and the nursing staff's reports in medical notes of children. Iran J Pedi. 2008;18(Suppl 1):33-40.

3. Cheraghi MA, Nikbakhat Nasabadi A, Mohammad Nejad E, Salari A, Ehsani Kouhi Kheyli S. Medication errors among nurses in intensive care units (ICU). J Mazand Univ Med Sci. 2012;21:115-9.

4. Biranvand S, Valizadeh F, Hosseinabadi R, Safari M. Disclosing medical errors and its relationship to disclosure of actual and hypothetical errors: nursing staff's attitude. J Med Ethics Hist Med. 2014;7(1):53-64.

5. Bucknall TK. Medical error and decision making: Learning from the past and present in intensive care. Aust Crit Care. 2010;23(3):150-6. doi: 10.1016/j.aucc.2010.06.001. [PubMed: 20594866].
6. Mohammadfam I, Movafagh M, Soltanian A, Salavati M, Bashirian S. Identification and evaluation of human errors among the nurses of coronary care unit using cream techniques. Int J Occup Saf Ergon. 2014;2(1):27-35.

7. Rogers AE, Dean GE, Hwang WT, Scott LD. Role of registered nurses in error prevention, discovery and correction. Qual Saf Health Care. 2008;17(2):117-21. doi:10.1136/qshc.2007.022699. [PubMed: 18385405].

8. Rothschild JM, Hurley AC, Landrigan CP, Cronin JW, Martell-Waldrop $\mathrm{K}$, Foskett C, et al. Recovery from medical errors: the critical care nursing safety net.Jt Comm J Qual Patient. 2006;32(2):63-72.

9. David G, Gunnarsson CL, Waters HC, Horblyuk R, Kaplan HS. Economic measurement of medical errors using a hospital claims database. Value Health. 2013;16(2):305-10. doi:10.1016/j.jval.2012.11.010.[PubMed: 23538182].

10. Freund Y, Goulet H, Bokobza J, Ghanem A, Carreira S, Madec D, et al. Factors associated with adverse events resulting from medical errors in the emergency department: two work better than one. J Emerg Med. 2013;45(2):157-62. doi: 10.1016/j.jemermed.2012.11.061. [PubMed: 23433610].

11. Hurley AC, Rothschild JM, Moore ML, Snydeman C, Dykes PC, Fotakis S. A model of recovering medical errors in the coronary care unit. Heart Lung. 2008;37(3):219-26. doi: 10.1016/j.hrtlng.2007.06.002. [PubMed: 18482634].

12. Kalra J, Kalra N, Baniak N. Medical error, disclosure and patient safety: a global view of quality care. Clin Biochem. 2013;46(13-14):1161-9. doi: 10.1016/j.clinbiochem.2013.03.025. [PubMed: 23578740].

13. Taft L. Apology and medical mistake: opportunity or foil. Annals Health L. 2005;14:55.

14. Caspi A, Rozenfeld V, Kleyman J. Prevention of medication errors in the hospital setting: the role of pharmacy students. Pharmacol Ther. 2005;30:183-6.

15. Najafpour J, Bromandfar S, Zahire M. Safety evaluation of patients in public hospitals under the guise of Medical Sciences, according to the World Health Organization protocols to assess patient safety. J Case Manag. 2014;5(1):47-58.

16. Saghiri S. Investigating errors of nursing and develop strategies to reduce errors in nurses. J Arch Mil Med. 2010;2:35-40.

17. Camire E, Moyen E, Stelfox HT. Medication errors in critical care: risk factors, prevention and disclosure. CMAJ. 2009;180(9):936-43. doi: 10.1503/cmaj.080869.

18. Garrouste-Orgeas M, Timsit JF, Vesin A, Schwebel C, Arnodo P, Lefrant JY, et al. Selected medical errors in the intensive care unit: results of the IATROREF study: parts I and II. Am J Respir Crit Care Med. 2010;181(2):134-42. doi: 10.1164/rccm.200812-18200C. [PubMed: 19875690].

19. Nasiri A, Babatabar HD, Mortazavi Y. Medication calculation skills of nurses in the intensive care unit. Crit Care Nurse. 2009;2(3):113-5.

20. Landrigan CP, Rothschild JM, Cronin JW, Kaushal R, Burdick E, Katz $\mathrm{JT}$, et al. Effect of reducing interns' work hours on serious medical errors in intensive care units. N Engl J Med. 2004;351(18):1838-48. doi: 10.1056/NEJMoa041406. [PubMed: 15509817].

21. Ryan C, Ross S, Davey P, Duncan EM, Francis JJ, Fielding S, et al. Prevalence and causes of prescribing errors: the PRescribing Outcomes for Trainee Doctors Engaged in Clinical Training (PROTECT) study. PLoS One. 2014;9(1):e79802. doi: 10.1371/journal.pone.0079802. [PubMed: 24404122].

22. Kang EK, Lihm HS, Kong EH. Association of intern and resident burnout with self-reported medical errors. Korean J Fam Med. 2013;34(1):36-42. doi: 10.4082/kjfm.2013.34.1.36. [PubMed: 23372904].

23. Sanchez AMM. Nature and frequency of prescription modifications: An evaluation from the community pharmacy. Afr J Pharm Pharmacol. 2014;8(8):240-5. doi: 10.5897/ajpp2012.1500.

24. Hannawa AF. Disclosing medical errors to patients: effects of nonverbal involvement. Patient Educ Couns. 2014;94(3):310-3. doi: 10.1016/j.pec.2013.11.007. [PubMed: 24332933]. 
25. Henneman EA, Gawlinski A, Blank FS, Henneman PL, Jordan D, McKenzie JB. Strategies used by critical care nurses to identify, interrupt, and correct medical errors. Am J Respir Crit Care Med. 2010;19(6):500-9.

26. Moosazadeh M, Yegane MR, Aghaei I, Mahmoodi S, Mehrzad F, Amiresmaili MR. Medical adverse events during patient's hospitalization at training Hospitals in Kerman. J Holist Nurs. 2014;24(2):68-76.

27. Mostafazadeh F, Asadzadeh-Monir F, Rhostamneghad M. Methods of patient safety education formedical group students. J Obstet Gynecol. 2013;8(1):23-9.

28. Kuhpayehzadeh J, Soltani Arabshahi SK, Bigdeli S, Maryami F. Determine Attitudes of TUMS Residents towards Medical Errors Self-disclosure and Prevalent Factors affecting it (1391). RJMS. 2013;20(108):53-60.

29. Rothschild JM, Churchill W, Erickson A, Munz K, Schuur JD, Salzberg CA, et al. Medication errors recovered by emergency department pharmacists. Ann Emerg Med. 2010;55(6):513-21. doi: 10.1016/j.annemergmed.2009.10.012. [PubMed: 20005011].

30. Vazin A, Zamani Z, Hatam N. Frequency of medication errors in an emergency department of a large teaching hospital in southern Iran. Drug Healthc Patient Saf. 2014;6:179-84. doi: 10.2147/DHPS.S75223. [PubMed: 25525391].

31. Stasiak P, Afilalo M, Castelino T, Xue X, Colacone A, Soucy N, et al. Detection and correction of prescription errors by an emergency department pharmacy service. Cjem. 2014;16(03):193-206. [PubMed: 24852582].

32. Vessal G. Detection of prescription errors by a unit-based clinical pharmacist in a nephrology ward. Pharm World Sci. 2010;32(1):59-65. doi: 10.1007/s11096-009-9341-9. [PubMed: 19838816].

33. Vazin A, Delfani S. Medication errors in an internal intensive care unit of a large teaching hospital: a direct observation study. Acta Med Iran. 2012;50(6):425-32. [PubMed: 22837122].

34. Mohsenzadeh A, Rezapour SMB, Birjandi M. Frequency of medical errors in hospitalized children in Khorramabad Madani Hospital dur- ing six months in 2008. Yafteh. 2010;11(4):31-8.

35. Zeraatchi A, Talebian MT, Nejati A, Dashti-Khavidaki S. Frequency and types of the medication errors in an academic emergency department in Iran: The emergent need for clinical pharmacy services in emergency departments. J Res Pharm Pract. 2013;2(3):118-22. doi: 10.4103/2279-042X.122384. [PubMed: 24991618].

36. Kozer E, Scolnik D, Macpherson A, Keays T, Shi K, Luk T, et al. Variables associated with medication errors in pediatric emergency medicine. Pediatrics. 2002;110(4):737-42. [PubMed: 12359787].

37. Seden K, Kirkham JJ, Kennedy T, Lloyd M, James S, McManus A, et al. Cross-sectional study of prescribing errors in patients admitted to nine hospitals across North West England. BMJ Open. 2013;3(1):e002036. doi:10.1136/bmjopen-2012-002036.

38. Aala A, Tabrizi J, Ranjbar F, Vahdati SS, Mohammadi N. Frequency of Burnout, Sleepiness and Depression in Emergency Medicine Residents with Medical Errors in the Emergency Department. ABCmed. 2014;2(2):49-54.

39. Mousavi M, Khalili H, Dashti-Khavidaki S. Errors in fluid therapy in medical wards. Int J Clin Pharm. 2012;34(2):374-81. doi: 10.1007/s11096012-9620-8. [PubMed: 22392405].

40. Dabaghzadeh F, Rashidian A, Torkamandi H, Alahyari S, Hanafi S, Farsaei S, et al. Medication errors in an emergency department in a large teaching hospital in Tehran. IJPR. 2013;12(4):937. [PubMed: 24523775].

41. Evans S, Mullen P. Verbal orders drug therapy and prescription in a UK ICU setting. Crit Care. 2009;13(suppl1):482-646. doi: 10.1186/cc7646.

42. Osmon S, Harris CB, Dunagan WC, Prentice D, Fraser VJ, Kollef MH. Reporting of medical errors: an intensive care unit experience. Crit Care Med. 2004;32(3):727-33. [PubMed:15090954].

43. Rothschild JM, Landrigan CP, Cronin JW, Kaushal R, Lockley SW, Burdick E, et al. The Critical Care Safety Study: The incidence and nature of adverse events and serious medical errors in intensive care. Crit Care Med. 2005;33(8):1694-700. [PubMed: 16096443]. 